\title{
TRANSGRESSIONS, CHERN-SIMONS INVARIANTS AND THE CLASSICAL GROUPS
}

\author{
JAMES L. HEITSCH \& H. BLAINE LAWSON, JR.
}

\section{Introduction}

Generalizing the work in [6], J. Simons has defined a family of natural transformations from principal bundles with connection over a manifold $M$ to characters on the integral cycles of $M$. Provided that certain Pontryagin forms vanish identically, these characters determine $R / Z$ cohomology classes, which for the tangent frame bundle of a riemannian manifold constitute a set of obstructions to conformally immersing the manifold into euclidean space.

In this paper we give a short proof that the Simons characters associated to any biinvariant metric connection on a compact Lie group $G$ are identically zero. At the same time we are able to compute the Chern-Simons invariants in the frame bundle of $G$ and by using the more delicate obstructions in [6] obtain nonimmersion theorems for certain group manifolds.

The paper is organized as follows. In $\S 2$ we summarize relevant facts from [6] and [9]. In $\S 3$ we reduce the problem of computing the Chern-Simons invariants to that of computing the map induced on cohomology by the adjoint representation. In $\S 4$ we apply these results to classical groups.

\section{Transgression classes and Chern-Simons theory}

The material in this section is presented without proof. For details see [6] and [9].

We begin by fixing notation. Let $G$ be a Lie group with Lie algebra $\mathfrak{g}$, and denote by $I^{*}(G)$ the graded ring of adjoint-invariant multilinear functions on $g$.

Denote by $E$ a principal $G$-bundle over a manifold $M$, and let $\theta$ be a connection on $E$ with curvature $\Omega$. To any $P \in I^{k}(G)$ we associate a real-valued $2 k$-form $P(\Omega, \cdots, \Omega)$ on $E$. This form is closed, horizontal and invariant, and hence it projects to a closed form on $M$. The element in $H^{2 k}(M ; R)$ so determined is independent of the connection $\theta$, and is therefore an invariant of the bundle $E$.

While $P(\Omega, \cdots, \Omega)$ may project to something nontrivial in the cohomology of $M$, it is always exact on $E$. In fact, if we set

Communicated by S. S. Chern, January 19, 1973. The first author was partially supported by NSF Grant No. GP-34785X, and the second author by NSF Grant No. GP29698 and the Sloan Foundation. 


$$
\Omega_{t}=t \Omega+\frac{1}{2}\left(t^{2}-t\right)[\theta, \theta]=d\left(\theta_{t}\right)+\frac{1}{2}\left[\theta_{t}, \theta_{t}\right],
$$

where $\theta_{t}=t \theta$, and define the transgression form $T P(\theta)$ of $P$ by

$$
T P(\theta)=k \int_{0}^{1} P\left(\theta, \Omega_{t}, \cdots, \Omega_{t}\right) d t,
$$

then we have $d(T P(\theta))=P(\Omega, \cdots, \Omega)$. Thus, if $P(\Omega, \cdots, \Omega) \equiv 0$, then $T P(\theta)$ determines an $R$ cohomology class on $E$, denoted $\{T P(\theta)\}$, which is in general not independent of $\theta$. For connections associated to riemannian metrics the theorem is the following

Theorem 2.1 (Chern-Simons [6, Theorem 4.5]). Let $E$ be the bundle of tangent frames on a manifold $M$, and suppose that $\theta$ and $\theta^{\prime}$ are the Levi-Civita connections of conformally related riemannian metrics $g$ and $g^{\prime}$ on $M$. Then for all $P \in I^{*}\left(G L_{n}\right)$ such that $P(\Omega) \equiv 0$, we have $P\left(\Omega^{\prime}\right) \equiv 0$ and

$$
\{T P(\theta)\}=\left\{T P\left(\theta^{\prime}\right)\right\} \text {. }
$$

Recall that the ring $I^{*}\left(G L_{n}\right)$ is generated by classes $p_{1}, \cdots, p_{[n / 2]}$, where degree $p_{j}=2 j$, with the following property. If we let $\tilde{p}_{j}$ be the $j$-th integral Pontrjagin class of $E$, and let

$$
r: H^{*}(M ; Z) \rightarrow H^{*}(M ; R)
$$

be the natural map, then the projection of $p_{j}(\Omega, \cdots, \Omega) \in r\left(\tilde{p}_{j}\right)$. For our purposes we need to consider also the inverse Pontryagin forms coming from polynomials $p_{j}^{\frac{1}{j}}$ given by the following identity in $t$ :

$$
\left(\sum_{j \geq 0} p_{j} t^{j}\right)\left(\sum_{j \geq 0} p_{j}^{\frac{1}{j}} t^{j}\right) \equiv 1
$$

where $p_{0}=p_{0}^{\perp}=1$. We say that $P \in I^{*}\left(G L_{n}\right)$ is integral if it is mapped to $H^{*}\left(B G L_{n} ; Z\right)$ by the Weil homomorphism. The $p_{j}^{\perp}$ are integral polynomials.

Before stating the next results we shall make a notational convention. For any space $X$, we denote by $\tilde{H}^{k}(X ; Z)$ the image of $H^{k}(X ; Z)$ in $H^{k}(X ; R)$ under the coefficient homomorphism.

Theorem 1.2 (Chern-Simons [6, Theorem 5.14]). Let $M$ be a compact oriented riemannian manifold of dimension $n$. Let $\theta$ denote the riemannian connection on the bundle of frames $E$. Suppose $M$ admits a conformal immersion in $\boldsymbol{R}^{n+k}$. Then for all $j$ with $[k / 2]+1 \leq j \leq[n / 2]$

1) $p \frac{1}{j}(\Omega, \cdots, \Omega) \equiv 0$,

2) $\left\{\frac{1}{2} T p_{j}^{\perp}(\theta)\right\} \in \tilde{H}^{4 j-1}(E ; Z)$.

Corollary 2.3. Let $M, E, \theta$ be as above, and suppose $\sigma: M \rightarrow E$ is a crosssection. Then a necessary condition that $M$ admit a conformal immersion in $\boldsymbol{R}^{n+k}$ is that $\sigma^{*}\left(\left\{\frac{1}{2} T p \frac{1}{j}(\theta)\right\}\right) \in \tilde{H}^{4 j-1}(M ; Z)$ for all $j$ with $[k / 2]+1 \leq j \leq$ $[n / 2]$. 
Suppose now that $\pi: E \rightarrow M$ is a principal $G L_{n}$ bundle with connection $\theta$ and curvature $\Omega$. To every integral $P \in I^{2 k}\left(G L_{n}\right)$, Simons associates a homomorphism $S P(\theta): Z_{4 k-1}(M) \rightarrow \boldsymbol{R} / \boldsymbol{Z}$ where $Z_{4 k-1}(M)$ denotes the integral $(4 k-1)$-cycles. If $P(\Omega, \cdots, \Omega) \equiv 0$, then $S P(\theta)$ defines a cohomology class $\{S P(\theta)\} \in H^{4 k-1}(M ; R / Z)$. For trivial principal bundles this class is related to $\{T P(\theta)\}$ as follows. Let $\sigma: M \rightarrow E$ be any cross-section, and let $\rho: H^{4 k-1}(M ; R)$ $\rightarrow H^{4 k-1}(M ; R / Z)$ be the natural reduction $\bmod Z$. Then

$$
\{S P(\theta)\}=\rho \circ \sigma^{*}(\{T P(\theta)\}) .
$$

We conclude this section by recalling the transgression map for compact Lie groups. Let $G$ be such a group, $B G$ its classifying space, and $\pi: E G \rightarrow B G$ the universal principal $G$ bundle over $B G$. The transgression map is a mapping $\tau: H^{k}(B G ; \Lambda) \rightarrow H^{k-1}(G ; \Lambda)$ for $k \geq 1$, where $\Lambda$ is some coefficient ring, defined as follows. Let $\gamma \in H^{k}(B G ; \Lambda)$ and choose $c \in \gamma$. The cocycle $\pi^{*} c$ is exact in $E G$ since $E G$ is contractible. Hence there is a cochain $c^{\prime}$ on $E G$ such that $\delta c^{\prime}=\pi^{*} c$. Then $i^{*} c^{\prime}$ is a cocycle in $G$ where $i: G \rightarrow E G$ is the fiber inclusion. The cohomology class $\tau(c)=\left\{i^{*} c^{\prime}\right\}$ is independent of the choices involved because of the acyclicity of $E G$.

We will need the following facts about the transgression map. The reference is Borel [2].

Lemma 2.4. Suppose $f: G \rightarrow G^{\prime}$ is a smooth homomorphism, and $B f: B G$ $\rightarrow B G^{\prime}$ is the induced map. Then the following diagram commutes:

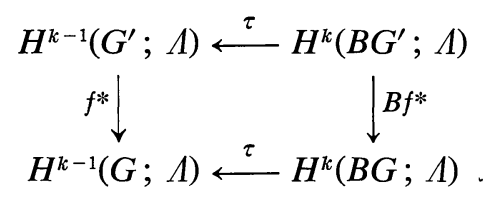

The map $\tau$ is not a homomorphism, but it does have the following relationship to the multiplicative structure in cohomology.

Lemma 2.5. $\tau$ maps primitive elements to primitive elements, and maps products to zero.

For $G=G L_{n}$ we have the following special result (cf. [4]).

Lemma 2.6. Let $p_{j}, p_{j}^{\perp} \in H^{4 j}\left(B G L_{n} ; \boldsymbol{R}\right)$ be the classes discussed above. Then

$$
\frac{1}{2} \tau p_{j}=-\frac{1}{2} \tau p_{j}^{\perp} \in \tilde{H}^{4 j-1}\left(G L_{n} ; Z\right),
$$

and, in fact, these classes are generators in the group

$$
\tilde{H}^{4 j-1}\left(G L_{n} ; Z\right) \text {. }
$$

Over the real numbers the transgression map can be given explicitly in terms of de Rham cohomology. Let $E \rightarrow M$ be any principal $G$-bundle over a 
manifold $M$ with connection $\theta$. Let $P \in I^{k}(G)$. We may consider $P$ as an element in $H^{2 k}(B G ; R)$ via the Weil homomorphism. Notice that the form $T P(\theta)$ defined in (2.1), when restricted to the fiber $G \subset E$, is closed, since $\left.d T P(\theta)\right|_{G}=$ $\left.P(\Omega, \cdots, \Omega)\right|_{G}=0$. The de Rham class of $\left.T P(\theta)\right|_{G}$ is exactly $\tau P \in H^{2 k-1}(G ; R)$. (Consider $E$ embedded in the universal bundle $E G$.) A straightforward calculation now shows that

$$
{ }_{\tau} P=\left(-\frac{1}{2}\right)^{k-1} \frac{k !(k-1) !}{(2 k-1) !} P\left(\omega_{G},\left[\omega_{G}, \omega_{G}\right], \cdots,\left[\omega_{G}, \omega_{G}\right]\right),
$$

where $\omega_{G}$ is the Maurer-Cartan form on $G$.

Note that the form in (2.2) is biinvariant and therefore harmonic if $G$ is compact.

\section{Compact Lie groups}

Let $G$ be a compact Lie group of dimension $n$ with Lie algebra $\mathfrak{g}$, and denote by $\langle\cdot, \cdot\rangle$ a biinvariant riemannian metric on $G .(\langle\cdot, \cdot\rangle$ corresponds to an $\mathrm{Ad}_{G}$-invariant inner-product in g.) Let $\theta$ be the associated riemannian connection on the frame bundle of $G$, and let $\Omega$ denote its curvature. Following Chern we shall adopt the convention that for $P \in I^{k}(G), P\left(\alpha_{2}, \alpha_{2}, \cdots, \alpha_{l}\right)$ for $l \leq k$ means that the argument $\alpha_{l}$ is repeated in the final $n-l$ slots. For example, $P(\Omega)=P(\Omega, \cdots, \Omega)$.

Lemma 3.1. For any $P \in I^{k}\left(G L_{n}\right), P(\Omega) \equiv 0$.

Proof. Let $\pi: E \rightarrow G$ denote the frame bundle of $G$, and consider the section

$$
\sigma: G \rightarrow E
$$

given by choosing an orthonormal basis of left-invariant vector fields. From Lemma 3.4 below we have

$$
\sigma^{*} \Omega=-\frac{1}{2} \sigma^{*}([\theta, \theta])
$$

and therefore

$$
\sigma^{*} P(\Omega)=\left(-\frac{1}{2}\right)^{k} \sigma^{*} P([\theta, \theta])
$$

Using the adjoint-invariance of $P$ (see $[5,(65)]$ ), we have

$$
P([\theta, \theta])=(k-1) P(\theta,[\theta[\theta, \theta]],[\theta, \theta]) .
$$

Since $[\theta,[\theta, \theta]] \equiv 0$ and $\pi^{*} \sigma^{*} P(\Omega)=P(\Omega)$, we have $P(\Omega) \equiv 0$.

It follows from Lemma 3.1 that for each integral form $P \in I^{k}\left(G L_{n}\right)$ there are classes $\{T P(\theta)\} \in H^{2 k-1}(E ; R)$ and $\{S P(\theta)\}=\rho \cdot \sigma^{*}\{T P(\theta)\} \in H^{2 k-1}(G ; \boldsymbol{R} / Z)$ defined. Our first main result is the following. 
Theorem 3.2. Let Ad: $G \rightarrow G L_{n}$ be the adjoint representation, and let $\theta$ be the Levi-Civita connection of a biinvariant metric on $G$. Then for any integral form $P \in I^{k}\left(G L_{n}\right)$ we have

$$
\sigma^{*}\{T P(\theta)\}=\operatorname{Ad}^{*}\left(\frac{1}{2} \tau P\right) .
$$

In particular, $\{S P(\theta)\}=0$.

The result that $\left\{S_{p_{i}^{\perp}}(\theta)\right\}=0$ for $G=S O(k)$ and all $i$ is also due to $\mathrm{J}$. Simons.

Proof. Let $e_{1}, \cdots, e_{n}$ be an orthonormal basis of left-invariant vector fields on $G$, and let $\omega_{1}, \cdots, \omega_{n}$ be the dual 1-forms. Then $\omega_{G}=\sum_{i=1}^{n} e_{i} \otimes \omega_{i}$ is the Maurer-Cartan form on $G$. The cross-section $\sigma: G \rightarrow E$ of the frame-bundle is given by $\sigma(g)=\left(g ; e_{1}(g), \cdots, e_{n}(g)\right)$ for $g \in G$. We recall that the connection $\theta$ is a $\mathrm{gl}_{n}$-valued 1 -form on $E$. Thus $\sigma^{*} \theta$ is a $\mathrm{gl}_{n}$-valued 1 -form on $G$. Our first step will be to compute this form.

Let $\mathrm{Ad}_{*}$ denote the differential of the adjoint transformation, and let ad: $\mathfrak{g}$ $\rightarrow \mathrm{gl}_{n}$ be the associated Lie algebra homomorphism.

Lemma 3.3. $\sigma^{*} \theta=\frac{1}{2} \operatorname{ad} \circ \omega_{G}=\frac{1}{2} \omega_{G L} \circ \mathrm{Ad}_{*}$.

Proof. The second equality follows immediately from the fact that ad $=$ $\mathrm{Ad}_{*}$ at the identity of $G$ and that $\mathrm{Ad}$ is a $C^{\infty}$ homomorphism.

To establish the first equality we need only to compute $\left(\sigma^{*} \theta\right)_{i j}(X)=$ $\left\langle e_{i}, V_{X} e_{j}\right\rangle$ for $1 \leq i, j \leq n$ where $\nabla_{X}$ is covariant differentiation with respect to the vector field $X$ on $G$ (see [7, p. 44] and [5]). For left-invariant vector fields $X$ and $Y$, covariant differentiation for a biinvariant metric is given by

$$
\nabla_{X} Y=\frac{1}{2}[X, Y]=\frac{1}{2} \operatorname{ad}_{X} Y,
$$

(see [8, pp. 113-114]). It follows that

$$
\left(\sigma^{*} \theta\right)_{i j}=\sum_{k=1}^{n}\left\langle e_{i}, \frac{1}{2} \operatorname{ad}_{e_{k}} e_{j}\right\rangle \omega_{k}=\left\langle e_{i}, \frac{1}{2} \operatorname{ad}_{\omega_{G}} e_{j}\right\rangle,
$$

that is, $\sigma^{*} \theta=\frac{1}{2}$ ad $\circ \omega_{G}$ as claimed.

Let $\Omega$ denote the curvature of the connection $\theta$. Recall that $\Omega$ is a $\mathrm{gl}_{n}$-valued 2-form.

Lemma 3.4.

$$
\sigma^{*} \Omega=\sigma^{*}\left(-\frac{1}{2}[\theta, \theta]\right)=-\frac{1}{2} \operatorname{Ad}^{*}\left(\left[\omega_{G L}, \omega_{G L}\right]\right) .
$$

Proof. By definition, $\Omega=d \theta+\frac{1}{2}[\theta, \theta]$. However, $\sigma^{*} d \theta=d \sigma^{*} \theta=$ $d\left(\operatorname{Ad}^{*}\left(\frac{1}{2} \omega_{G L}\right)\right)=\operatorname{Ad}^{*}\left(\frac{1}{2} d \omega_{G L}\right)=\operatorname{Ad}^{*}\left(-\left[\frac{1}{2} \omega_{G L}, \frac{1}{2} \omega_{G L}\right]\right)$ (by the Maurer-Cartan equations $)=-\left[\frac{1}{2} \mathrm{Ad}^{*} \omega_{G L}, \frac{1}{2} \mathrm{Ad}^{*} \omega_{G L}\right]=-\left[\sigma^{*} \theta, \sigma^{*} \theta\right]=-\sigma^{*}[\theta, \theta]$.

We can now complete the proof of the theorem. Recall that

$$
T P(\theta)=k \int_{0}^{1} P\left(\theta, \Omega_{t}\right) d t
$$

where 


$$
\Omega_{t}=t \Omega+\frac{1}{2}\left(t^{2}-t\right)[\theta, \theta]
$$

Thus

$$
\begin{aligned}
\sigma^{*} T P(\theta) & =k \int_{0}^{1} P\left(\sigma^{*} \theta, \sigma^{*} \Omega_{t}\right) d t \\
& =k \int_{0}^{1} P\left(\operatorname{Ad}^{*} \frac{1}{2} \omega_{G L},\left(\frac{1}{2} t^{2}-t\right) A d^{*}\left[\frac{1}{2} \omega_{G L}, \frac{1}{2} \omega_{G L}\right]\right) \\
& =\operatorname{Ad}^{*}\left(P\left(\omega_{G L},\left[\omega_{G L}, \omega_{G L}\right]\right) k \int_{0}^{1}\left(\frac{1}{2}\right)^{2 k-1}\left(\frac{1}{2} t^{2}-t\right)^{k-1} d t\right) .
\end{aligned}
$$

Using the fact that

$$
\int_{0}^{1} t^{r}(1-t)^{s} d t=\frac{r ! s !}{(r+s+1) !}
$$

and (2.2), we have

$$
\begin{aligned}
\sigma^{*}\{T P(\theta)\} & =\operatorname{Ad}^{*}\left[\frac{1}{2}\left(-\frac{1}{2}\right)^{k-1} \frac{k !(k-1) !}{(2 k-1) !} P\left(\omega_{G L},\left[\omega_{G L}, \omega_{G L}\right]\right)\right] \\
& =\operatorname{Ad}^{*}\left(\frac{1}{2} \tau P\right) .
\end{aligned}
$$

This proves (3.2).

The fact that $\{S P(\theta)\}=0$ follows from (3.2), Lemmas 2.5 and 2.6, and the fact (cf. [2]) that $\tilde{H}^{*}\left(B G L_{n} ; Z\right)$ is generated as a ring by the classes $p_{j}$. This completes the proof of Theorem 3.2.

We would now like to use Corollary 2.3 to obtain results on the nonexistence of conformal immersions. Theorem 3.2 reduces this problem to computing the map

$$
\mathrm{Ad}^{*}: H^{*}\left(G L_{n} ; Z\right) \rightarrow H^{*}(G ; Z)
$$

modulo torsion. In particular we are interested in this map on the elements $\frac{1}{2} \tau p \frac{1}{j}=-\frac{1}{2} \tau p_{j}$.

To simplify matters in the following discussion, we shall replace $G L_{n}$ with the subgroup $0(n)$. Since Ad is an orthogonal representation and $B O(n)$ has the homotopy-type of $B G L_{n}$, everything remains essentially unchanged.

Let $G$ be an $n$-dimensional compact Lie group, and $\mathrm{Ad}: G \rightarrow 0(n)$ its adjoint representation, and suppose $T_{G} \subset G$ is a maximal torus. Consider the following commutative diagram (cf. Lemma 2.4):

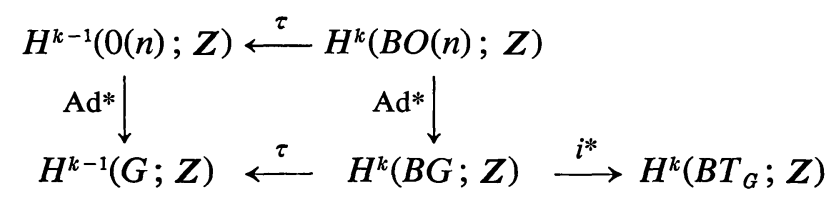


where $\tau$ denotes the transgression map, and $i^{*}$ is the map induced by inclusion. We are interested in computing the left vertical map. When $G$ is a classical group, the maps $\tau$ are well known (cf. [2]), and it will suffice to compute the right vertical map. Since the map $i^{*}$ is injective modulo torsion, and for the classical groups the images of $i^{*}$ are well-known, we need in fact only to compute the map $i^{*} \circ \mathrm{Ad}^{*}$. This is accomplished by computing the weights of the adjoint representation.

Specifically, we proceed as follows. Let $G$ be a compact $n$-dimensional Lie group, and $\rho: G \rightarrow O(n)$ a homomorphism, and choose maximal tori $T_{G} \subset G$ and $T_{0} \subset 0(n)$ so that $\rho\left(T_{G}\right) \subset T_{0}$. Let $\hat{\omega}_{1}, \cdots, \hat{\omega}_{[n / 2]} \in H^{1}\left(T_{G} ; Z\right)$ denote the weights of the representation (the images under $\rho^{*}$ of canonical generators for $H^{1}\left(T_{0} ; Z\right)$ ), and $\omega_{1}, \cdots, \omega_{[n / 2]}$ their preimages in $H^{2}\left(B T_{G} ; Z\right)$ under transgression. We recall that $\tau: H^{2}\left(B T_{G} ; Z\right) \rightarrow H^{1}\left(T_{G} ; Z\right)$ is an isomorphism, and $H^{*}\left(B T_{G} ; Z\right)$ is a polynomial algebra on 2-dimensional generators. If $p=1+$ $p_{1}+p_{2}+\cdots+p_{[n / 2]}$ is the universal total Pontrjagin class in $H^{*}(B O(n) ; Z)$, then a theorem of Borel-Hirzebruch gives us the following

Theorem 3.5 ([3, Theorem 10.3]).

$$
i^{*} \rho^{*}(p)=\prod_{j=1}^{[n / 2]}\left(1+\omega_{j}^{2}\right)
$$

where $i^{*}: H^{*}(B G ; Z) \rightarrow H^{*}\left(B T_{G} ; Z\right)$ is the natural map.

Ultimately we wish to know if $i^{*} \rho^{*}(p)$ is an even class in $i^{*}\left(H^{*}(B G ; Z)\right)$. For this we need a precise knowledge of the image, which can be had from the fact that $i^{*}\left(H^{*}(B G ; Z)\right.$ is the subring of $H^{*}\left(B T_{G} ; Z\right)$ invariant by the induced action of the Weyl group.

\section{Computations for the classical groups}

We shall now carry out the computations just indicated for the case where $G$ is a compact classical Lie group. We begin by recalling some basic facts concerning these groups (cf. [1], [2], [4]). Suppose $G$ has rank $k$, and let $i: T_{G} \subset G$ denote a maximal torus. In what follows $\theta_{1}, \cdots, \theta_{k}$ will denote a set of generators for $H^{1}\left(T_{G} ; Z\right)$, and will also denote the corresponding elements in $H^{2}\left(B T_{G} ; Z\right)$. All of our computations will take place in the group $\tilde{H}^{*}(B G ; Z) \cong i^{*} H^{*}(B G ; Z) \subset H^{*}\left(B T_{G} ; Z\right)$.

Let $G=S O(2 k)$. Then the weights of the adjoint representation are $\theta_{i}+\theta_{j}$ and $\theta_{i}-\theta_{j}$ for $1 \leq i<j \leq k$. Hence, by (3.5),

$$
i^{*} \mathrm{Ad}^{*} p=\prod_{i<j}\left(1+\left(\theta_{i}-\theta_{j}\right)^{2}\right)\left(1+\left(\theta_{i}+\theta_{j}\right)^{2}\right) .
$$

The ring $\tilde{H}^{*}(B G ; Z)$ is generated by the classes

$$
P_{j}=\sigma_{j}\left(\theta_{1}^{2}, \cdots, \theta_{k}^{2}\right) \quad \text { for } j=1, \cdots, k,
$$


and $\chi=\theta_{1} \cdots \theta_{k}$ with $\chi^{2}=P_{k}$. The class $\frac{1}{2} \tau P_{j}, 1 \leq j \leq k-1$, is a generator of $\tilde{H}^{4 j-1}(S O(2 k) ; Z)$, and $\tau P_{k}=\tau \chi^{2}=0 . \sigma_{j}$ is the $j$-th symmetric function.

Let $G=S O(2 k+1)$. Then the weights of the adjoint representation are $\theta_{i}+\theta_{j}, \theta_{i}-\theta_{j}$ for $1 \leq i<j \leq k$ and $\theta_{i}$ for $1 \leq i \leq k$. Hence

$$
i^{*} \mathrm{Ad}^{*} p=\prod_{i<j}\left(1+\left(\theta_{i}-\theta_{j}\right)^{2}\right)\left(1+\left(\theta_{i}+\theta_{j}\right)^{2}\right) \prod_{i}\left(1+\theta_{i}^{2}\right) .
$$

The ring $\tilde{H}^{*}(B G ; Z)$ is generated by the classes

$$
P_{j}=\sigma_{j}\left(\theta_{1}^{2}, \cdots, \theta_{k}^{2}\right), \quad j=1, \cdots, k,
$$

and $\frac{1}{2} \tau P_{j}$ is a generator in $\tilde{H}^{4 j-1}(G ; Z)$

Let $G=U(k)$. Then the weights of the adjoint representation are $\theta_{i}-\theta_{j}$ for $1 \leq i<j \leq k$. Hence

$$
i^{*} \mathrm{Ad}^{*} p=\prod_{i<j}\left(1+\left(\theta_{i}-\theta_{j}\right)^{2}\right) .
$$

The ring $H^{*}(B G ; Z)$ is generated by the classes

$$
C_{j}=\sigma_{j}\left(\theta_{1}, \cdots, \theta_{k}\right) \quad \text { for } j=1, \cdots, k .
$$

Finally, the class $\tau C_{j}$ is a generator of $H^{2 j-1}(U(k) ; Z)$.

Let $G=S p(2 k)$. Then the weights of the adjoint representation are $\theta_{i}-\theta_{j}$, $\theta_{i}+\theta_{j}$ for $1 \leq i<j \leq k$ and $2 \theta_{i}$ for $1 \leq i \leq k$. Hence

$$
i^{*} \operatorname{Ad}^{*} p=\prod_{i<j}\left(1+\left(\theta_{i}-\theta_{j}\right)^{2}\right)\left(1+\left(\theta_{i}+\theta_{j}\right)^{2}\right) \prod_{i}\left(1+4 \theta_{i}^{2}\right) .
$$

The ring $\tilde{H}^{*}(B G ; Z)$ is generated by the classes

$$
P_{j}=\sigma_{j}\left(\theta_{1}^{2}, \cdots, \theta_{k}^{2}\right) \quad \text { for } j=1, \cdots, k,
$$

and the class $\frac{1}{2} \tau P_{j}$ is a generator of $\tilde{H}^{4 j-1}(S p(2 k) ; Z)$.

Because the transgression map sends nontrivial products to zero, we need only to compute $\mathrm{Ad}^{*} p$ modulo products in $\tilde{H}^{*}(B G ; Z)$. The results are summarized in the following table:

\begin{tabular}{|c|c|}
\hline$G$ & $\begin{array}{c}\mathrm{Ad}^{*} p_{l} \text { mod products in } \widetilde{H}^{*}(B G ; Z) \\
1 \leq l \leq \frac{1}{2} n\end{array}$ \\
\hline$S O(n)$ & $\left(n-2^{2 l-1}\right) P_{l}$ \\
\hline$U(n)$ & $(-1)^{l} 2 n C_{2 l}$ \\
\hline$S p(n)$ & $\left(n+2^{2 l-1}\right) P_{l} \quad(n$ even $)$ \\
\hline
\end{tabular}

In each case above, $\mathrm{Ad}^{*} p_{l}$ must be a sum of nontrivial products for $l>[n / 2]$, and for $S O(2 k), \mathrm{Ad}^{*} p_{k} \equiv 0 \bmod$ products. 
This information combined with Theorem 3.2 and Corollary 2.3 gives

Theorem 4.1. $S O(2 k+1)$ with a biinvariant metric does not conformally immerse in euclidean space (or the sphere) in codimension $2 k-1$.

Proof. $\frac{1}{2} \sigma^{*}\left\{T p_{k}(\theta)\right\}=\frac{1}{4} \tau \mathrm{Ad}^{*} p_{k}=\frac{1}{2}\left(2 k+1-2^{2 k-1}\right) \frac{1}{2} \tau P_{k} \equiv \frac{1}{4} \tau P_{k} \neq 0$ modulo integral classes. Apply Corollary 2.3.

Theorem 4.2. $U(2 k+1)$ with a biinvariant metric does not conformally immerse in euclidean space in codimension $2 k-1$.

Proof. $\frac{1}{2} \sigma^{*}\left\{T p_{k}(\theta)\right\}=\frac{1}{4} \tau \mathrm{Ad}^{*} p_{k}=(-1)^{k} \frac{1}{2}(2 k+1) \tau C_{2 k} \equiv \frac{1}{2} \tau C_{2 k} \neq 0$ modulo integral classes.

Note that by the theorem of Hirsch both $S O(n)$ and $U(n)$ immerse in euclidean space in codimension one. These immersions cannot be conformal for $n$ odd. These results generalize a result of Chern and Simons [6] for $S O(3)$.

Observing that the projection $\pi: G \rightarrow G / \Gamma$ is a local isometry when $\Gamma$ is a discrete subgroup and $G / \Gamma$ is given the induced metric, we have

Corollary 4.1. Let $\Gamma \subset G$ be a discrete subgroup where $G=S O(2 k+1)$ or $G=U(2 k+1)$. Then $G / \Gamma$ with a metric induced from a biinvariant metric on $G$ does not conformally immerse in euclidean space in codimension $2 k-1$.

We would like similar results for the spaces $G / \Gamma$ where $G=S O(2 k), U(2 k)$, or $S p(2 k)$ and $\Gamma$ is a discrete subgroup. We have the following general result.

Theorem 4.3. Let $G$ be a compact Lie group, and $\Gamma$ a discrete subgroup. If $\frac{1}{4} \mathrm{Ad}^{*}\left(\tau p \frac{\perp}{j}\right)$ does not contain a $Z$ cochain invariant under the action of $\Gamma$, then $G / \Gamma$ with a metric induced from a biinvariant metric on $G$ does not conformally immerse in euclidean space in codimension $2 j-1$.

Proof. Let $\pi: G \rightarrow G / \Gamma$ be the projection map, and observe that $\gamma \in H^{*}(G / \Gamma ; R)$ represents a $Z$ class iff $\pi^{*}(\gamma)$ contains a $Z$ cochain invariant under the action of $\Gamma$. Let $\theta$ and $\hat{\theta}$ be the Levi-Civita connections on $G$ and $G / \Gamma$ respectively, and $\sigma$ the cross-section of the tangent frame bundle of $G$ as before. Let $\hat{\sigma}$ be the corresponding cross-section for $G / \Gamma$. Then

$$
\pi^{*}\left(\hat{\sigma}^{*}\left\{\frac{1}{2} T p_{j}^{\perp}(\theta)\right\}\right)=\sigma^{*}\left(\frac{1}{2}\left\{T p_{j}^{\perp}(\theta)\right\}\right)=\frac{1}{4} \operatorname{Ad}^{*}\left(\tau p_{j}^{\perp}\right) .
$$

Apply Corollary 2.3.

Proof of the formulas given in table. In the following discussion $\sigma_{j}$ and $S_{j}$ will denote respectively the $j$-th symmetric function and the $j$-th power sum of the variables indicated; i.e., $S_{j}\left(X_{1}, \cdots, X_{p}\right)=\sum_{i=1}^{p} X_{i}^{j}$. We recall (cf. [10, p. 81]) that

$$
S_{l}-S_{l-1} \sigma_{1}+S_{l-2} \sigma_{2}-\cdots+(-1)^{l-1} S_{1} \sigma_{l-1}+(-1)^{l} l \sigma_{l}=0 .
$$

Consider the case $U(k)$. Since $\sigma_{l}\left(\theta_{1}, \cdots, \theta_{k}\right)=C_{l}$ and $S_{l}\left(\theta_{1}, \cdots, \theta_{k}\right) \in$ $\boldsymbol{R}\left[C_{1}, \cdots, C_{k}\right]$, we have from (4.5) that modulo products of $C_{i}$ 's

$$
S_{l}\left(\theta_{1}, \cdots, \theta_{k}\right) \equiv(-1)^{l+1} l C_{l} \text {. }
$$

We now set $y_{i j}=\left(\theta_{i}-\theta_{j}\right)^{2}$ for $1 \leq i<j \leq k$, and observe that by (4.3) 


$$
i^{*} \operatorname{Ad}^{*} p_{l}=\sigma_{l}\left(y_{i j}\right) .
$$

However, $\sigma_{l}\left(y_{i j}\right), S_{l}\left(y_{i j}\right) \in R\left[C_{1}, \cdots, C_{k}\right]$, so we have from (4.5) that modulo products of $C_{j}$ 's

$$
\begin{aligned}
\sigma_{l}\left(y_{i j}\right) & \equiv \frac{(-1)^{l+1}}{l} S_{l}\left(y_{i j}\right) \\
& =\frac{(-1)^{l+1}}{l} \sum_{i<j}\left(\theta_{i}-\theta_{j}\right)^{2 l} \\
& =\frac{(-1)^{l+1}}{2 l} \sum_{i, j=1}^{k}\left(\theta_{i}-\theta_{j}\right)^{2 l} \\
& =\frac{(-1)^{l+1}}{2 l} \sum_{i, j=1}^{k}\left(\theta_{i}^{2 l}-2 l \theta_{i}^{2 l-1} \theta_{j}+\left(\begin{array}{c}
2 l \\
2
\end{array}\right) \theta_{i}^{2 l-2} \theta_{j}^{2}+\cdots+\theta_{j}^{2 l}\right) .
\end{aligned}
$$

Using

$$
\sum_{i, j=1}^{k} \theta_{i}^{2 l-r} \theta_{j}^{r}=S_{2 l-r}\left(\theta_{1}, \cdots, \theta_{k}\right) S_{r}\left(\theta_{1}, \cdots, \theta_{k}\right)
$$

we have

$$
\sigma_{l}\left(y_{i j}\right) \equiv(-1)^{l+1} \frac{k}{l} S_{2 l}\left(\theta_{1}, \cdots, \theta_{k}\right) \equiv(-1)^{l} 2 k C_{2 l}
$$

Hence

$$
i^{*} \mathrm{Ad}^{*} p \equiv 1+2 k \sum_{l=1}^{k}(-1)^{l} C_{2 l}
$$

(Note that $i^{*} \operatorname{Ad}^{*} p_{j}$ is necessarily a sum of nontrivial products for $j>k$.)

We now consider $G=S O(2 k)$. Let $x_{i j}=\left(\theta_{i}+\theta_{j}\right)^{2}$ and $y_{i j}=\left(\theta_{i}-\theta_{j}\right)^{2}$ for $1 \leq i, j \leq k$. Then by (4.1),

$$
i^{*} \operatorname{Ad}^{*} p=\sigma_{l}\left(x_{i j}, y_{i j}\right) .
$$

Since $\sigma_{l}\left(x_{i j}, y_{i j}\right)$ and $S_{l}\left(x_{i j}, y_{i j}\right)$ are invariant under permutations of the $\theta_{i}$ 's and under each map $\theta_{i} \rightarrow-\theta_{i}$, they must both lie in the $\operatorname{ring} R\left[P_{1}, \cdots, P_{k}\right]$. Consequently, by (4.5) we have

$$
\begin{aligned}
(-1)^{l+1} \sigma_{l}\left(x_{i j}, y_{i j}\right) & \equiv \frac{1}{l} S_{l}\left(x_{i j}, y_{i j}\right) \\
& =\frac{1}{l} \sum_{i<j}\left(\theta_{i}+\theta_{j}\right)^{2 l}+\left(\theta_{i}-\theta_{j}\right)^{2 l} \\
& =\frac{1}{2 l}\left[\sum_{i, j=1}^{k}\left(\left(\theta_{i}+\theta_{j}\right)^{2 l}+\left(\theta_{i}-\theta_{j}\right)^{2 l}\right)-\sum_{i=1}^{k}\left(2 \theta_{i}\right)^{2 l}\right]
\end{aligned}
$$




$$
\begin{aligned}
& =\frac{1}{2 l}\left[2 \sum_{i, j=1}^{k}\left(\theta_{i}^{2 l}+\left(\begin{array}{c}
2 l \\
2
\end{array}\right) \theta_{i}^{2 l-2} \theta_{j}^{2}+\cdots+\theta_{j}^{2 l}\right)-2^{2 l} \sum_{i=1}^{k} \theta_{i}^{2 l}\right] \\
& \equiv \frac{1}{l}\left(2 k-2^{2 l-1}\right) S_{l}\left(\theta_{1}^{2}, \cdots, \theta_{k}^{2}\right) \\
& \equiv(-1)^{l+1}\left(2 k-2^{2 l-1}\right) P_{l} .
\end{aligned}
$$

Hence

$$
i^{*} \operatorname{Ad}^{*} p \equiv 1+\sum_{l=1}^{k}\left(2 k-2^{2 l-1}\right) P_{l}
$$

For $G=S O(2 k+1)$, we have from (4.2), (4.1) and (4.7) that

$$
\begin{aligned}
i^{*} \operatorname{Ad}^{*} p & \equiv\left(1+\sum_{l=1}^{k}\left(2 k-2^{2 l-1}\right) P_{l}\right)\left(1+\sum_{l=1}^{k} P_{l}\right) \\
& \equiv 1+\sum_{l=1}^{k}\left(2 k+1-2^{2 l-1}\right) P_{l} .
\end{aligned}
$$

The case $G=S p(k)$ is entirely similar.

We conclude with a few remarks. Since the weights of Ad are the roots of the Lie algebra of $G$, the computation of $\mathrm{Ad}^{*} p$ should be straightforward for the exceptional groups. The difficulty lies in knowing the map $\tau: \tilde{H}^{*}(B G ; Z)$ $\rightarrow \tilde{H}^{*}(G ; Z)$ exactly.

The above results carry over easily to products of $U(n), S O(n)$, and $S p(n)$.

It would be useful to compute the Chern-Simons invariants for left invariant metrics on compact Lie groups. However, this seems to be a difficult problem. The invariants might not even exist since a priori $P(\Omega)$ does not have to be identically zero. The connections in question are in $1-1$ correspondence with bilinear functions from $\mathfrak{g} \times \mathfrak{g}$ to $\mathfrak{g}$, where $\mathfrak{g}$ is the Lie algebra of the group. If $X$ and $Y$ are left invariant vector fields, the correspondence is given by $\alpha(X, Y)=\nabla_{X} Y$, and $\nabla$ is biinvariant iff $\alpha(X, X)=0$ for all $X \in \mathfrak{g}$. This is an extremely strong condition. It allowed us to conclude that $\nabla_{X} Y=\frac{1}{2} \operatorname{ad}_{X} Y$ which was the basic fact needed in our computation.

In the special case where the map $\alpha^{\prime}: \mathfrak{g} \rightarrow \mathfrak{g l}_{n}$ given by $\alpha^{\prime}(X)=\alpha(X,-)$ is a multiple, say $\varepsilon$, of a Lie algebra homomorphism, we have

$$
\Omega=\frac{\varepsilon-1}{2 \varepsilon}[\theta, \theta]
$$

and $P(\Omega) \equiv 0$ by invariance. The class $\{T P(\theta)\}$ depends strongly on $\alpha^{\prime}$. For example if $\alpha^{\prime} / \varepsilon$ is the differential of a Lie group homomorphism $A: G \rightarrow$ $G L(n, R)$, then for $P \in I^{k}\left(G L_{n}\right)$ 


$$
\sigma^{*}\{T P(\theta)\}=\frac{\beta(\varepsilon)}{\beta(1)} A^{*}(\tau P)
$$

where

$$
\beta(a)=\int_{0}^{a} t^{k-1}(1-t)^{k-1} d t
$$

In particular if $\varepsilon=\frac{1}{2}$, then

$$
\sigma^{*}\{T P(\theta)\}=A^{*}\left(\frac{1}{2} \tau P\right)
$$

as before.

\section{References}

[1] A. Borel, Sur la cohomologie des espaces fibrés principaux et des espaces homogènes de groupes de Lie compacts, Ann. of Math. 57 (1953) 115-207.

[2] _ Topics in the homology theory of fiber bundles, Springer, Berlin, 1967.

[ 3 ] A. Borel \& F. Hirzebruch, Characteristic classes and homogeneous spaces. I, Amer. J. Math. 80 (1958) 458-538.

[ 4 ] $\frac{\text { J }}{315}$ Characteristic classes and homogeneous spaces. II, Amer. J. Math. 81 (1959) 315-382.

[ 5 ] S. S. Chern, Geometry of characteristic classes, Proc. Thirteenth Biennial Seminar, Canadian Math. Congress, 1972, 1-40.

[6] S. S. Chern \& J. Simons, Characteristic forms and geometric invariants, Ann. of Math. 99 (1974) 48-69.

[7] S. Helgason, Differential geometry and symmetric spaces, Academic Press, New York, 1962.

[ 8 ] J. Milnor, Morse theory, Annals of Math. Studies, No. 51, Princeton University Press, Princeton, 1963.

[ 9 ] J. Simons, Characteristic forms and trangression. II: characters associated to a connection, preprint.

[10] B. L. Van der Waerden, Modern algebra, Vol. I, F. Ungar, New York, 1931.

UNiVERsity of CALIFornia, BERKeley. 